\title{
Iatrogenic Cushing's syndrome caused by treatment with traditional herbal medicine, a case report
}

\author{
Hari Hendarto \\ Faculty of Medicine and Health Sciences, \\ Islamic State University (UIN) Syarif Hidayatullah Jakarta \\ Department of Internal Medicine \\ Jakarta, Indonesia
}

\begin{abstract}
Iatrogenic Cushing's syndrome is caused by the administration of exogenous glucocorticoids. The use of exogenous glucocorticoids has been found as an adulterant in many traditional, alternative medicines. In this case report, we describe a patient with iatrogenic Cushing's syndrome due to chronic use of traditional herbal medicine. A 40-year-old man was diagnosed with iatrogenic Cushing's syndrome due to the long-term use of traditional herbal medicine. After that, he progressively was presented with sudden weight gain, moon faces, supraclavicular fat pads, buffalo hump, truncal obesity, purple striae, and hypertension. Initial investigations detected a low morning serum cortisol level and low serum adrenocorticotropin (ACTH). Other hormonal investigations of the hypothalamic-pituitary axis were normal. He was diagnosed as iatrogenic Cushing's syndrome due to long-term use of traditional herbal medicine that suspected to contain steroids. Physicians should be aware the importance of taking a medical history carefully, with special interest to the use of regular, traditional herbal medicine.
\end{abstract}

Keywords-Iatrogenic Cushing's syndrome; traditional herbal medicine

\section{INTRODUCTION}

Cushing's syndrome is the collection of signs and symptoms due to excessive glucocorticoids from either an exogenous or endogenous source. Iatrogenic (drug-related or exogenous) Cushing's syndrome, the most common form, is caused by administration of exogenous glucocorticoids, and will actually lead to increased cortisol levels inside the body [1]. The use of exogenous glucocorticoids has been found as an adulterant in many traditional, alternative medicines. ${ }^{2}$ In Indonesia, the use of traditional herbal medicine is common among those with musculoskeletal problems. In this case report, we describe a patient with iatrogenic Cushing's syndrome due to chronic use of traditional herbal medicine. In Indonesia, the use of traditional herbal medicine is common among those with musculoskeletal problems. In this case report, we describe a patient with iatrogenic Cushing's syndrome due to chronic use of traditional herbal medicine.

\section{CASE SUMMARY}

A 40-year-old man was admitted in hospital for complaint of petechia in both legs. In a past medication history interview, he stated that for at least one year, muscles throughout his body were often sore, and since then he had been ingesting traditional herbal medicine. Upon ingestion, his muscle soreness was gone and eventually he became addicted to the said traditional herbal medicine. Following this, he progressively was presented with rapid weight gain from 50 to $80 \mathrm{~kg}$ in 6 months. Neuropsychological disturbances such as depression and emotional irritability were also observed. $\mathrm{He}$ was a non smoker and did not consume alcohol. He is not on any hormonal replacement therapy. There was no significant related disease in family history.

On general examination the patient was conscious and coherent, his blood pressure was 150/90 $\mathrm{mmHg}$. Examination of the eyes revealed no bilateral hemianopia, but the patient showed moon like faces and the buffalo humps. The examination of both cardiovascular and respiratory systems was unremarkable. Examination of the abdomen revealed fat accumulation on the trunk (central obesity), but there was no abdominal tenderness, hepatosplenomegaly or striae. Purple striae marks and purpura appeared in his abdominal and legs area. The possibility of Cushing's syndrome secondary to the traditional herbal medicine was considered.

The extent of laboratory investigations detected a low morning serum cortisol level $(35.6 \mathrm{ng} / \mathrm{ml}$, normal > 210 $\mathrm{ng} / \mathrm{ml}$ ) and low serum adrenocorticotropin (ACTH) level 3 $\mathrm{pg} / \mathrm{ml}$ (reference range: $5-20 \mathrm{pg} / \mathrm{ml}$ ), suggest that he had ACTH independent Cushing's syndrome. Adrenal insufficiency may occur due to prolonged hypothalamuspituitary-adrenal (HPA) axis suppression. The thyroid function test and other hormonal investigations of the hypothalamicpituitary axis were within normal limits. Chest radiography and an ultrasound of the abdomen was done, which showed no abnormality. 
He was diagnosed with iatrogenic Cushing's syndrome due to long-term use of traditional herbal medicine that is suspected to contain steroids. Unfortunately, he did not know exactly what the product contained. Moreover, the traditional herbal medicine was packaged in a bottle without any labels. He was informed of the adverse drug reactions secondary to consumption of his traditional herbal medicine. Sudden discontinuation of corticosteroids after prolonged use, could lead to adrenal insufficiency and adrenal crisis [2]. The traditional herbal medicine was then tapered down until it was totally stopped. Fortunately, the patient did not have any evidence of adrenal insufficiency as he discontinued the traditional herbal medicine. The patient condition improved after withdrawal of the traditional herbal medicine. Blood pressure came to normal $(130 / 80 \mathrm{~mm} \mathrm{Hg})$. His weight dropped $10 \mathrm{~kg}$ in two months and his red striation and petechia were also reduced. Most would require hormone replacement with hydrocortisone until recovery of the HPA function [2].

\section{DISCUSSION}

Cushing's syndrome, a disorder resulting from chronic exposure to excess glucocorticoids, was discovered by Harvey Cushing in 1912 [1]. Cushing syndrome occurs when a body has a higher than normal level of the hormone cortisol. This hormone is normally made in the adrenal glands. Corticotropin-releasing hormone (CRH) from the hypothalamus, transported via the hypothalamic-pituitary tract induces the anterior lobe of the pituitary gland to release ACTH into the systemic circulation, which, in turn, stimulates the adrenal cortex to synthesize and secrete glucocorticoids [2]. The traditional stigmata includes the classic moon face, the appearance of buffalo hump, weight gain, central and dorsocervical obesity, supraclavicular fat accumulation, and central obesity with redistribution of body fat to truncal areas $[1,3]$. Thinned skin with wide purple striae, myopathy, muscle weakness, poor wound healing, and increased incidence of infection can also be seen. The elevated level of cortisol causes hyperglycemia, osteoporosis, weakness, hypertension, increased susceptibility to infection, decreased resistance to stress, and mood swings [4]. Cushing's syndrome can be partitioned by whether the cause of increased cortisol secretion is ACTH dependent or ACTH independent. In the ACTH-dependent Cushing's syndrome form, the adrenal cortex has received excessive stimulation ACTH, leading to inappropriate and pathological adrenal cortisol secretion. The two sources of this excessive ACTH are an ACTH-secreting pituitary adenoma and ectopic (non-pituitary) ACTH secretion, usually due to a neuroendocrine tumour [4]. In the ACTH-independent Cushing's syndrome form, circulating glucocorticoid activity is increased without ACTH stimulation. In endogenous ACTH-independent Cushing's syndrome, the adrenal glands produce cortisol autonomously without ACTH due to the either an adrenal tumor or be iatrogenic (drug-related or exogenous) [4]. Endogenous Cushing's syndrome is traditionally classified as ACTH dependent, when pathologic ACTH secretion drives cortisol production, or as ACTH independent, when the adrenal glands autonomously secrete excessive cortisol [1]. Exogenous or iatrogenic Cushing's syndrome is more common than endogenous Cushing's syndrome and results from the administration of supraphysiologic doses of glucocorticoids.

Oral corticosteroids therapy is the most common cause of iatrogenic Cushing's syndrome, but some studies have been published about adrenal insufficiency after treatment with intra-articular steroid injection, topical, or dermal steroid therapy, and after inhaled corticosteroids. However, literature about adrenal insufficiency after ingestion of oral, herbal, or alternative mixtures is very scarce [5]. Traditional herbal medicines have been used extensively by patients for many pain disorders such as chronic musculoskeletal problems. There are many reasons why people choose to use traditional herbal medicines [6]. The side effects of prescription drugs are widely publicized, whereas herbal preparations are perceived as natural, mild and safe, and therefore harmless [7]. Although the majority of them are safe, some traditional herbal medicines carry risks. The majority of traditional herbal medicines have two or more adulterants. Among the common adulterants in herbal medicine are corticosteroids and nonsteroidal anti-inflammatory drugs [2]. However, the use of corticosteroids is not without risk, side effects include musculoskeletal effects such as osteoporosis and risk of pathological fracture [8]. Corticosteroids affect behavior and mood with the risk of depression. It also causes peptic ulcer disease, immunosuppression, and ophthalmogical problems such as glaucoma and cataracts [2].

Iatrogenic Cushing's syndrome is the result of prolonged administration of exogenous corticosteroid administration in large amounts [1]. Through the HPA feedback mechanism, iatrogenic Cushing's leads to adrenal suppression and low endogenous cortisol levels [9]. In most cases of exogenous Cushing's syndrome, the morning serum cortisol is found to be remarkably low, ACTH levels also should be relatively low, as pituitary production will be suppressed by exogenous steroids [3]. The suppression of ACTH leads to atrophy of the adrenal cortex. Adrenal suppression should be anticipated in any patient taking the equivalent of $7.5 \mathrm{mg}$ prednisolone / day for over three weeks, because it may be months or many years until the adrenal is awakened again [9]. Not all cases of exogenous Cushing's syndrome comes from prescribed or therapeutic use of glucocorticoids, as within this case illustrated by an unfortunate event which has resulted from the use of traditional herbal as described before. Because traditional herbal medicine is not commonly considered to have glucocorticoid activity, physicians may not be aware of the associated risks.

\section{CONCLUSION}

Presence of corticosteroids in traditional herbal drugs can lead to iatrogenic Cushing's syndrome. Physicians should be aware the importance of taking medical history carefully, with special interest to the use of regular traditional herbal medicine. Presence of corticosteroids in traditional herbal medicine can cause severe systemic side effects like Cushing's syndrome and other potentially dangerous side effects. Physicians have a role 
to counsel and educate patients as consumers, when using alternative medicine.

\section{REFERENCES}

[1] N.A. Wagner-Bartak, A. Baiomy, S.V. Mukhi, A.C. Morani, B.R. Korivi, S.G. Waguespack, K.M. Elsayes,"Cushing Syndrome: Diagnostic Workup and Imaging Features, With Clinical and Pathologic Correlation," Am. J. Roentgenol. vol.209, pp.19-32, 2017.

[2] S.G. Sazlina, A Zaiton, "Cushing's syndrome secondary to adulterated complementary and alternative medicine," Malays. Fam. Physician. vol.4, pp.94-97, 2009.

[3] R.L Hopkins, M.C. Leinung, "Exogenous Cushing's syndrome and glucocorticoid withdrawal," Endocrinol. Metab. Clin. North. Am. vol.34, pp.371-384, 2015.

[4] G Gottiganti, J.K. Badhvel, G.R Giri Dornadula, A.K Petam, B.C. Pothugunt, "Case report on dexamethasone induced iatrogenic cushing syndrome,” Int. J. Harm. Sci. Rev. Res. vol.45, pp.151-152, 2017.
[5] P.C. Oldenburg-Ligtenberg, M.M.L. van der Westerlaken, “A woman with Cushing's syndrome after use of an Indonesian herb: a case report.” J.M. vol.65, pp.150-152, 2007.

[6] H. Hendarto, F.R. Sari, A. Muqorrobin, C. Ahmad, E. Amelia, Hermansyah, L. Respati, N. Maulida, "Cinnamomun Cassia extract improves blood glukcose and lipid profile in aloxan - induced diabetic rats," Asian Jr. Of Microbiol. Biotech. Env. Sc. vol.17, pp.409414, 2015.

[7] C.M. Corns, "Herbal remedies and clinical biochemistry," Ann. Clin. Biochem. vol.40, pp.489-507, 2003.

[8] H. Hendarto, "Parathyroid Adenoma in a Young Female Presenting," vol. 49, pp.69-73, 2017

[9] P. Steward. "Iatrogenic adrenal suppression: diagnosis and management," Endocrine. Abstracts. vol.19, pp.S60, 2009. 